\title{
La reputación en la imagen de marca de la cerveza en México: Un análisis exploratorio (Brand reputation on brand image of beer in Mexico: An exploratory analysis)
}

\section{Osiris María Echeverría Ríos` Arturo Tavizón Salazar*}

\begin{abstract}
Reputation of brands has become a relevant aspect for companies, thanks to the consumer purchasing decision. The purpose of this research is to identify the effect of the Reputation on the Brand Image of beer in Mexico. To achieve the objective, a quantitative research was designed, and a questionnaire was applied in the state of Tamaulipas (Mexico) to 329 consumers. The methodology used for the statistical treatment of the data is through a linear regression through the SPSS version 21. The result indicates a positive effect of the reputation on the image of the beers of the Mexican market, significant at 0.01 by consumers of the region.
\end{abstract}

Key words: beer, brand image, brand reputation

JEL: M310, M370.

Resumen. La reputación de las marcas se ha convertido en un aspecto relevante para las empresas, gracias a la decisión de compra de los consumidores. El propósito de esta investigación es identificar el efecto de la Reputación en la Imagen de Marca de la cerveza en México. Para cumplir el objetivo se diseñó una investigación cuantitativa y se aplicó un cuestionario en el estado de Tamaulipas (México) a 329 consumidores. La metodología utilizada para el tratamiento estadístico de los datos es mediante una regresión lineal por medio del SPSS versión 21. El resultado indica un efecto positivo de la reputación en la imagen de las marcas de cervezas del mercado mexicano significativo al 0.01 por parte de los consumidores de la región.

Palabras clave: cerveza, imagen de marca, reputación

\footnotetext{
- Universidad Politécnica Metropolitana de Hidalgo, Hidalgo, México, C. P. 43680. Email: cposirisecheverria@gmail.com

* Universidad Autónoma de Nuevo León, Facultad de Contaduría Pública y Administración, San Nicolás de los Garza, N.L., México. Email: arturo.tavizonslz@uanl.edu.mx
} 


\section{Introducción}

En la época contemporánea las empresas han convertido a las marcas en un importante activo intangible. En consecuencia, para la industria de bebidas alcohólicas supone un reto mantener la imagen de sus marcas y su percepción en un lugar decisivo en la mente del consumidor. Al ser una valuación meramente subjetiva y al involucrar productos de bajo riesgo de compra para el consumidor, como la cerveza.

Por ello, se considera útil investigar la industria de la cerveza en México. Hay que señalar que, a pesar de ser un país con una economía emergente, es un principal exportador y consumidor de este producto, con el sexto lugar a nivel mundial y que está presente en más de 180 países en todo el mundo (Cerveceros de México, 2015). Por consiguiente, para esta industria, se consideran las marcas nacionales de México con una ventaja competitiva.

Sin embargo, se debe mencionar que existen escasos estudios que aborden la relación causal de la reputación en la percepción de imagen de marca (Alvarado y Schlensiger, 2008, Aranda, Gómez y Molina, 2015, Nguyen y Leblanc, 2001, Veloutsou y Moutinho, 2009). De manera similar en lo relacionado con las investigaciones realizadas en México, hasta el momento no se han encontrado estudios empíricos que se hayan realizado con dichas variables.

Por lo precedente, el propósito de esta investigación, es identificar la influencia que tiene la reputación de marca en la percepción de imagen de marca de los clientes de bebidas alcohólicas, en específico de la cerveza. Por lo que se realizó un estudio cuantitativo exploratorio, mediante un cuestionario, para posteriormente analizarse por medio del SPSS, versión 21. Por lo tanto, se ha formulado una hipótesis que tratará de contrastar la percepción de imagen de marca que tienen las personas sobre su marca de cerveza preferida.

La investigación se estructura por secciones. En la siguiente sección se presenta el marco teórico relacionado con las variables independiente y dependiente estudiadas. Posteriormente se desarrolla el apartado metodológico y se analizan los resultados. Finalmente, se exponen las principales aportaciones al conocimiento. 


\section{Imagen de marca}

La imagen de marca es un concepto difícil de abordar debido a sus múltiples definiciones y enfoques. Esta cuestión es analizada por Dobni y Zinkhan (1990) y Zinkhan y Hirschheim (1992) para quienes ha sido uno de los problemas tópicos desde la introducción del concepto en 1950. A pesar del avance de las investigaciones y de que existen más estudios empíricos, esta realidad no ha cambiado como lo sugiere Stern, Zinkhan y Jaju (2001) y más recientemente Malik, Naeem y Munawar (2012) y Lee, James y Kim (2014).

No obstante, el presente estudio se basa en la categoría de elementos sicológicos/cognitivos donde se establece la teoría de valor de marca en función a la imagen de marca de acuerdo a Keller (1993). De este modo Keller (2001) conceptualiza la imagen de marca en términos como asociaciones de marca, rendimiento de marca, imaginación de marca, juicios del consumidor y sentimientos del consumidor. Lo que implica que el valor de marca es definido desde la perspectiva de un consumidor individual y su concepto es encuadrado y proveído por lo que los consumidores saben acerca de las marcas y que el conocimiento implica estrategias de mercadotecnia (Keller, 1993).

En este orden de ideas, los modelos encontrados para su medición de acuerdo a Cian (2011) y Malik et al. (2012) se categorizan desde los unidimensionales hasta los multidimensionales. Por su parte, Martínez y Pina (2009) aportan que solo existen dos variables incluidas en imagen de marca, simplificándolas en imagen funcional y reputación de marca. Dicho modelo fue replicado por Hariri y Vazifehdust (2011) quienes consideran que la reputación se encuentra dentro de la imagen afectiva. Ellos comprueban que en su modelo hay dos dimensiones, la imagen funcional y la afectiva, a diferencia de Martínez y Pina (2009). Por otra parte, Aranda et al. (2015) concuerdan con el modelo propuesto por Martínez y Pina (2009) al establecer la imagen funcional y la reputación como dos variables, no obstante, hay un marcado contraste al utilizar una variable global de imagen de marca además de la imagen funcional y la reputación en su modelo a diferencia de sus predecesores.

En cuanto al tipo de producto analizado el modelo de Martínez et al. (2005) se ha probado en lácteos-queso (Pascual, Puleva, Ram y Clesa), calzado deportivo-botas de esquí (Adidas, Nike, Kelme, Kappa), electrónica- 
teclado musical, cámaras digitales, maletas (Sony, Philips, Thomson y Grunding). Mientras que el analizado por Martínez y Pina (2009) en pasta dental (Colgate), lentes (Signal), esquís (Nike y Puma) y Dvd, cursos online y seguros (Amena y Movistar).

En consecuencia, la escala desarrollada por Martínez y Pina (2009) es la misma que ha sido probada por Aranda et al. (2015) en bebidas alcohólicas, específicamente en vino. Y más recientemente por Echeverría y Medina (2016) quienes probaron la escala en los vehículos compactos en México (imagen funcional, afectiva y reputación). Por lo anterior, se optó por este modelo para el presente estudio. Estas escalas han sido válidas para aplicarlas en los estudios de la imagen de marca de un producto, como lo observa Cian (2011).

\section{Reputación}

La reputación es un concepto abstracto, según Marquina-Feldman, ArrellanoBahamonde y Velasquez-Bellido (2014) la contemplan como una construcción a través del tiempo o un componente histórico, dicho concepto da relativamente más estabilidad y brinda una naturaleza duradera, al contrario del constructo de imagen. Mientras que para Barnett, Jermier y Lafferty (2006) el concepto se ha usado intercalado con imagen e identidad.

De esta manera, existe un marcado contraste entre la escuela relacional de Chun (2005) y lo propuesto por Gotsi y Wilson (2001) que introducen la reputación desde dos escuelas diferentes: la análoga y la diferenciada, la primera conforma la imagen y la reputación corporativa como conceptos idénticos e intercambiables (Gotsi y Wilson, 2001), mientras que la diferenciada explica que la imagen y la reputación son conceptos considerados distintos, aunque relacionados, como menciona también Alvarado Herrera (2008).

Por lo que autores como Alvarado y Schlesinger (2008) retoman la imagen de marca y reputación como dos variables por separado al momento de analizar los servicios de telefonía celular en Valencia, España siguiendo la teoría de Ahearne, Bhattacharya y Gruen (2005). De la misma manera que Christou (2003) en su estudio del sector hotelero en Inglaterra, Grecia y Alemania determinan que existe una interdependencia entre ambas variables 
coincidiendo con la escuela diferenciada de Gotsi y Wilson (2001) y Pérez y Rodríguez del Bosque (2014).

En tanto que Orozco y Roca (2011) mencionan que la imagen de marca y la reputación han sido utilizadas como sinónimos (Capriotti, 2009). Lo que es similar a lo realizado por Martínez y Pina (2009) que toman en cuenta la reputación como una dimensión propia de la imagen de marca en su definición. Por lo tanto, para este último modelo una de las principales diferencias con los ítems de Alvarado y Schlesinger (2008), es que no integran a la calidad como parte esencial de ambas variables a diferencia de Aaker (1996) y Chen (1996).

Al profundizar y al no tener un solo criterio teórico al abordar la imagen y la reputación, las investigaciones relacionadas con la causalidad entre ambas variables como ya se planteó, son escasas y desde perspectivas variadas (Alvarado y Schlensiger, 2008, Aranda et al., 2015, Currás-Pérez, Bigné-Alcañiz y Alvarado-Herrera (2009), Nguyen y Leblanc, 2001, Nguyen et al. (2016) y Veloutsou y Moutinho (2009).

Nguyen y Leblanc (2001) argumentan con su estudio que hay una significante interdependencia entre imagen y reputación, al menos en el sector educativo, telecomunicación y ventas al detalle en Canadá. De este modo, prueban la relación moderadora entre una imagen corporativa y reputación corporativa. No obstante, existe un importante vacío de estudios en lo que respecta a la relación causal entre dichas variables.

Por su parte Veloutsou y Moutinho (2009) hacen referencia a la imagen de marca como un punto crítico en el desarrollo de las relaciones con la marca, argumentando que mientras más positiva sea la reputación de marca, será más fuerte la relación con la marca. Sin embargo, de acuerdo a su estudio empírico, aunque existe una relación significativa entre imagen y reputación, no es tan poderosa como la que hay entre tribalismo y las relaciones de marca, donde se incluye la imagen de marca.

En contraste para Alvarado y Schlensinger (2008) existe un efecto de la imagen en la reputación, desde esta perspectiva la imagen se observa que es medida como una única variable. La construcción de los ítems engloba la parte de imagen afectiva y funcional, como contemplan Martínez y Pina (2009). Al mismo tiempo, Currás-Pérez, Bigné-Alcañiz y Alvarado-Herrera (2009) proponen que existe una influencia directa y positiva entre la imagen 
de una corporación socialmente responsable sobre el prestigio de la marca, la cual forma parte de la construcción de la reputación de una empresa.

De esta mismo manera, Aranda et al. (2015) confirma que la imagen funcional y la reputación afectan a la imagen global. Lo que significa que la imagen funcional, la cual es un signo de la calidad del producto y el precio percibido, y que además refleja la identidad y las emociones de la región en donde el vino es producido (imagen afectiva) influyen en la imagen de marca de la denominación de origen.

Asimismo la reputación confirma que tiene un fuerte impacto sobre la región que produce vino. $Y$ por el contrario no encuentran un impacto significativo entre la imagen afectiva y la imagen global. En tanto que para Nguyen et al. (2016) en un estudio más reciente con las marcas de las instituciones educativas encuentran que existe un efecto entre la imagen de marca y la reputación de marca en particular en las universidades.

La problemática identificada radica en la dificultad de determinar el sentido de causa-efecto sobre las variables de imagen afectiva, reputación de marca e imagen de marca debido a las teorías planteadas por las escuelas análoga y diferencial. Una posible causa es la carencia de una definición uniforme de reputación de marca (Gotsi y Wilson, 2001, Chun, 2005, Barnett et al.,2006 y Marquina-Feldman et al., 2014), lo que se refleja en los puntos contrapuestos de las escuelas relacional, análoga y diferenciada que la estudian.

Por lo señalado, es pertinente proponer una hipótesis que contraste la opinión del consumidor de la reputación de una marca de cerveza en la imagen de marca:

H1. La reputación de marca tiene un efecto positivo y directo sobre la percepción de la imagen de marca de la cerveza en México.

\section{Método}

En el ámbito nacional no se encontró hasta el momento un estudio que revise con anterioridad el efecto de reputación de marca en la imagen de marca, por lo tanto, la investigación que se realizó es de carácter exploratorio. Al mismo tiempo se define como cuantitativa, con diseño no experimental, 
correlacional, causal y transeccional, al realizarse en un momento en el tiempo, por medio de un cuestionario del 1 de noviembre de 2017 al 15 de enero del 2018.

De esta manera la variable dependiente es la imagen de marca y la variable independiente la reputación de marca. Las cuales se operacionalizan de la siguiente manera:

- Variable dependiente: imagen de marca (la imagen de marca de la cerveza es identificada con el lugar a donde pertenece y la cerveza es asociada a una imagen de marca positiva).

- Variable independiente: reputación de marca (es de prestigio o de renombre, ocupa un lugar propio en la mente de los consumidores a diferencia de otra marca, infunde o impone respeto y es producida por una empresa socialmente responsable, denota el tipo de persona que soy, da una clara impresión del nivel socioeconómico de las personas que las consumen y despierta un sentimiento de apego, de afinidad o simpatía hacia ella).

Una vez definidas la hipótesis y variables, se construyó un cuestionario con un piloteo previo. Para medir estas dos variables se utilizó la escala Likert de 5 puntos, donde los individuos indican su percepción desde totalmente en desacuerdo (1) a totalmente de acuerdo (5). Esta escala fue considerada una elección ideal para el establecimiento del nivel de las percepciones de la variable imagen de marca (Cian, 2011). De esta forma se trata de replicar y mejorar las investigaciones anteriores para aplicarlo en México a la población con las siguientes características: personas mayores de 18 años, que tengan conocimientos sobre las marcas de cerveza.

Para la validación del instrumento, se consideró a académicos y expertos en su análisis, para posteriormente pilotearse con ayuda de 30 observaciones del perfil descrito. La última versión del instrumento comprendió para la imagen de marca: 2 preguntas y para reputación: 7 preguntas, lo cual se puede observar en la Tabla 1. Se agregó también cuestionamientos acerca de las variables sociodemográficas: edad, sexo, nivel o grado de estudios, ocupación y promedio del ingreso mensual que se percibe. 
Tabla 1. Operacionalización de imagen de marca y reputación

\begin{tabular}{lll}
\hline Variable & Items & Autores \\
\hline Imagen de Marca & La imagen de marca de la cerveza & Adaptado de: Martínez y Pina \\
& es identificada con el lugar a donde & (2009); Aranda et al. (2015) y \\
& pertenece. & Echeverría y Medina (2016) \\
& La cerveza es asociada a una & \\
& imagen de marca positiva. & \\
& Es de prestigio o de renombre. & \\
& Ocupa un lugar propio en la mente & Adaptado de: Ahearne et al. (2005), \\
Reputación de Marca & de los consumidores a diferencia & Alvarado y Schelinger (2008) y \\
& de otra marca. Infunde o impone & Martínez et al. (2005) y Echeverría \\
& respeto. & y Medina (2016) \\
& Es producida por una empresa & \\
& socialmente responsable. & \\
& Dice algo sobre el tipo de persona & \\
& que soy. & \\
& Da una clara impresión del nivel \\
& socioeconómico de las personas \\
& que la consumimos. \\
& Despierta un sentimiento de apego, \\
& de afinidad o simpatía hacia ella. & \\
\hline
\end{tabular}

Fuente: Elaboración propia a partir de Ahearne et al. (2005); Alvarado y Schelinger (2008); Aranda et al. (2015); Echeverría y Medina (2016) Martínez et al. (2005) y Martínez y Pina (2009).

La muestra se seleccionó a conveniencia, resultando 329 observaciones. Aplicándose en el estado de Tamaulipas en México en las universidades (Universidad Autónoma de Tamaulipas, Universidad Politécnica de Victoria y el Instituto Tecnológico de Victoria), oficinas de gobierno de Tamaulipas, en tiendas departamentales. De esta forma las personas respondieron de manera autoadministrada, anónima y confidencial.

Posteriormente con la información recolectada, se realizó la descripción general e inferencial. En primer lugar, se realizó el análisis de confiabilidad de las escalas con el alfa de Cronbach. Una vez hecho esto, la técnica estadística utilizada para contrastar la hipótesis planteada fue el análisis de regresión lineal, mediante el software SPSS versión 21.

\section{Resultados}

Una vez capturados los datos, se realizó el análisis con el software SPSS. Inicialmente, se realizó el análisis descriptivo donde se determinó que de los encuestados $51.5 \%$ son y en cuanto a las mujeres representaron el $48.5 \%$. Son personas de diversas edades con un rango desde los 18 hasta los 76 
años. De este modo el $52.3 \%$ de las personas encuestadas se concentran de los 18 a los 25 años; es decir, personas jóvenes con un alto apego a la tecnología y por ende con información actualizada para la toma de decisiones.

En tanto que el nivel o grado de estudios se identificó en licenciatura con un $37.2 \%$ y un $22.2 \%$ en preparatoria, mientras que el promedio de ingresos es el $67.3 \%$ y se encuentra entre menos de 1,000 a 2,999 pesos. Por último, en la muestra el $36.5 \%$ de las personas encuestadas son empleadas del sector público y privado y $41.5 \%$ son estudiantes.

Al iniciar la inferencia estadística primero se realizó el análisis de confiabilidad del instrumento de recolección de datos por medio del Alfa de Cronbach como medida de la consistencia interna de las variables que debe ser igual o superior a 0.7 (Nunnally, 1978), no obstante, para las primeras fases de la investigación un valor de fiabilidad de 0.6 o 0.5 puede ser suficiente (Nunnally, 1967). Así mismo, $R^{2}$ indica el coeficiente de determinación, así como la varianza explicada por la variable independiente y su relación con la dependiente dentro del modelo, la cual de acuerdo con Chin (1998) debe alcanzar al menos un valor de a 0.1 porque valores menores, aun siendo significativos, proporcionan poca información.

De este modo se obtuvo que la reputación de marca sobre pasa los mínimos recomendados de 0.7 de Alfa de Cronbach (0.894). Mientras que la imagen de marca está dentro de lo suficiente al ser un estudio de carácter exploratorio (0.693) de Alfa de Cronbach. Posterior a esto se realizó la regresión lineal, llamada también "modelo de regresión lineal de dos variables 0 modelo de regresión lineal bivariada, debido a que en este modelo se relacionan las dos variables X y Y" (Wooldridge, 2015, p.22), como se muestra a continuación (1):

donde

$$
Y=\beta_{0}+\beta 1 x+\varepsilon
$$

$Y=$ Imagen de Marca

$\beta 1 x=$ Reputación

$\varepsilon=$ Error

En la Tabla 2 se observa el resumen del modelo causal. Se determina entonces que la Hipótesis es aceptada $\left(R=0.764, R^{2}=0.584\right.$ y $p<0.01=99 \%$ de 
confianza). Con una beta de 0.766 y un error de estimación de 0.64669872 . Esto determina que la reputación de marca tiene una influencia positiva en la imagen de marca de la cerveza, la cual es identificada en el lugar a donde pertenece y la cerveza es asociada a una imagen de marca positiva.

Tabla 2. Resumen modelo causal

\begin{tabular}{ccccc}
\hline Modelo & $\mathbf{R}$ & R cuadrado & $\begin{array}{c}\text { R cuadrado } \\
\text { corregida }\end{array}$ & $\begin{array}{c}\text { Error típico de la } \\
\text { estimación }\end{array}$ \\
\hline 1 & $0.764 a$ & 0.584 & 0.583 & 0.64669872 \\
\hline
\end{tabular}

Fuente: Elaboración propia a partir del SPSS versión 21

Nota a: Variables predictoras: (Constante), Reputación

En la Tabla 3 se muestra que los intervalos de confianza no contemplan la posibilidad de tener el valor de cero, por lo tanto, la variable reputación de marca es causal de la imagen de marca y la cual explica el $58 \%$ de la variabilidad de la imagen de marca.

Tabla 3. Coeficientes del modelo causal

\begin{tabular}{cccccccc}
\hline & $\begin{array}{c}\text { Coeficientes } \\
\text { estandarizados }\end{array}$ & \multicolumn{3}{c}{$\begin{array}{c}\text { Coeficientes } \\
\text { tipificados }\end{array}$} & \multicolumn{3}{c}{$\begin{array}{c}\text { Intervalo de confianza de 95\% } \\
\text { para B }\end{array}$} \\
\hline Modelo & $\mathrm{B}$ & $\begin{array}{c}\text { Error } \\
\text { típ. }\end{array}$ & Beta & $\mathrm{t}$ & Sig. & Límite inferior & $\begin{array}{c}\text { Límite } \\
\text { superior } \\
1\end{array}$ \\
$\begin{array}{c}1 \\
\text { (constante) }\end{array}$ & -.003 & 0.036 & & -0.097 & 0.923 & -0.074 & 0.067 \\
Regr factor & 0.766 & 0.036 & 0.764 & 21.309 & 0.000 & 0.695 & 0.836 \\
\hline
\end{tabular}

Fuente: Elaboración propia a partir del SPSS versión 21

\section{Discusión}

En México, a pesar de ser uno de los principales exportadores de cerveza, en los estudios realizados hasta el momento no se ha revisado la relación entre la reputación y la imagen de marca. Por este motivo, el objetivo fue determinar la influencia que tiene la reputación como punto primordial en las estrategias de ventaja competitiva en mercadotecnia, y obtener así evidencia empírica exploratoria de la relación causal entre ambas variables.

En esta investigación de carácter exploratoria en la que se demuestra que si existe un efecto significativo al $99 \%$ de confiabilidad de la reputación 
de marca con respecto a su causalidad en la imagen de marca. Los intervalos de confianza demuestran la causalidad entre ambas variables, así como la varianza explicada en un $58 \%$ por el modelo siguiente:

$Y($ imagen de marca $)=-0.003+0.766 \times 1$ (reputación de marca $)$

El modelo anterior significa que por cada unidad de cambio en la reputación de marca percibido por el consumidor la imagen de marca aumentará en 0.766 unidades de percepción del consumidor. Lo que contribuye a enfocar esfuerzos y estrategias de mercadotecnia que contribuyan al incremento de la reputación de marca para contribuir al incremento en la percepción y valor intangible de la imagen de marca. Siendo esta la principal aportación del modelo.

De esta forma para el consumidor mexicano la imagen de la marca es beneficiada por su reputación de marca, tiene un lugar en la mente del cliente, el respeto que infunde, si se relaciona de manera emocional con dicha marca y además que tiene un sentido claro del nivel socioeconómico de las personas que consumen la marca. Esto se puede deber a que las marcas mexicanas conservan una reputación a nivel internacional que afecta su imagen y que ha conservado a través del tiempo.

En relación al contexto estudiado, las investigaciones realizadas a nivel internacional con autores como Aranda et al. (2015), Currás-Pérez y Alvarado-Herrera (2009) y en menor medida Veloutsou y Moutinho (2009) coinciden con los resultados obtenidos al existir una relación directa entre ambas variables. Por otro lado, se hace mención de Alvarado y Schlensiger (2008) y Nguyen et al. (2016) de modo similar, a pesar que su estudio lo realiza con la imagen como la variable independiente y la reputación la dependiente.

En cuanto a la teoría propuesta es indispensable mencionar que los ítems que forman parte de la variable reputación conforman también la imagen afectiva, a diferencia de lo que proponen Aranda et al. (2015) y Martínez y Pina (2009) y Echeverría y Medina (2016), quienes determinan la reputación y la imagen afectiva por separado. De esta forma se observa que en la región central de Tamaulipas la reputación refleja también el sentido de la imagen afectiva en el consumidor. 
Por último, al ser un instrumento cuya opinión depende de la marca de cerveza de preferencia, no se puede distinguir qué marca especifica fue elegida por los participantes, por lo que los resultados pueden divergir entre las marcas. Además, en cuanto a la escala se debe realizar un estudio a mayor profundidad de los ítems que engloba la variable reputación y su relación con la imagen afectiva.

Es indispensable trabajar con herramientas estadísticas multivariantes y contemplar la percepción que los consumidores tienen de las distintas marcas de cerveza.

\section{Referencias}

Aaker, D. A. (1996). Measuring brand equity across products and markets, California Management Rev., 38(Spring), 102-120.

Ahearne, M., Bhattacharya, C. B. \& Gruen, T. (2005). Antecedents and Consequences of Customer-Company Identification: Expanding the Role of Relationship Marketing, Journal of Applied Psychology, 90(3), 574-585.

Alvarado-Herrera, A. (2008). Responsabilidad social empresarial percibida desde una perspectiva sostenicéntrica, y su influencia en la reputación de la empresa y en el comportamiento del turista. Tesis doctoral. Universitat de Valéncia en España.

Alvarado, H. A. \& Schelesinger, D. M. W. (2008). Dimensionalidad de la responsabilidad social empresarial percibida y sus efectos sobre la imagén y la reputación: Una aproximación desde el modelo de Carroll, Estudios gerenciales, 24(108), 37-59.

Aranda, E., Gómez, M. \& Molina, A. (2015). Consumers' brand images of wines, British Food Journal, 8(117), 2057-2077.

Barnett, M. L, Jermier, J. M. \& Lafferty, B. A. (2006). Corporate reputation: The definitional landscape, Corporate Reputation Review, 9(1), 26-38.

Bloemer, J., Ruyter, K. \& Peeters, P. (1998). Investigating drivers of bank loyalty: the complex relationship between image, service quality and satisfaction, International Journal of Bank Marketing, 16(7), 276-286.

Capriotti, P. P. (2009). Branding Corporativo. España: Andros Impresores.

Cerveceros de México. (2015). La cerveza mexicana. http://www.cervecerosdemexico.org.mx/ la-cerveza-mexicana/. Recuperado: Agosto 13,2015

Chen, A. C. H. (1996). The measurement and building of customer-based brand equity. Tesis doctoral. National Chengchi University in Taiwan.

Christou, E. (2003). Guest Loyalty Likelihood in Relation to Hotels' Corporate Image and Reputation: A Study of Three Countries in Europe, Journal of Hospitality and Leisure Marketing, 10(3-4), 25-56. 
Chin, W. W. (1998). Issues and opinion on structural equation modeling, MIS Quarterly, 2(2): vii-xvi.

Chun, R. (2005). Corporate reputation: Meaning and measurement, International Journal of Management Reviews, 7(2), 91-109.

Cian, L. (2011). How to measure brand image: a reasoned review, The Marketing Review, 11(2), 165-187.

Currás-Pérez, R., Bigné-Alcañiz, E. \& Alvarado-Herrera, A. (2009). The role of selfdefinitional principles in consumer identification with a socially responsible company, Journal of Business Ethics, 89, 547-564.

Dobni, D. \& Zinkhan, G.M. (1990). In search of Brand image: a foundation analysis, Advances in Consumer Research, 17(1), 110-119.

Echeverría, R. O. \& Medina, Q. M. (2016). Imagen de marca en la percepción de la calidad del consumidor de los vehículos compactos, Investigación Administrativa, 45(117), 722.

Gotsi, M. \& Wilson, A.M. (2001). Corporate reputation: Seeking a definition, Corporate Communications: An International Journal, 6(1), 24-30.

Hariri, M. \& Vazifehdust, H. (2011). How does brand extension affect brand image? 2010 International Conference on Business and Economics Research. Conferencia llevada a cabo en Kuala Lumpur, Malaysia.

Keller, K. L. (1993). Conceptualizing, measuring and managing customer-based brand equity, Journal of Marketing, 57(1), 1-22.

Keller, K. L. (2001). Building customer-based brand equity: A blueprint for creating strong brands, Marketing Management, 3(15), 15-19.

Lee, J. L., James, J. D. \& Kim, Y. K. (2014). A Reconceptualization of brand image, International Journal of Business Administration, 5(4), 1-11.

Malik, M.E., Naeem, B. y Munawar, M. (2012). Brand image: Past, present and future, Journal of Basic and Applied Scientific Research, 2(12), 13069-13075.

Martinez, E., Montaner, T. \& Pina, J. (2005). Propuesta de medición de la imagen de marca: un análisis aplicado a las extensiones de marca, Revista Asturiana de Economía RAE, 33, 89-112.

Martínez, E. \& Pina, J. (2009). Modeling the brand extensions influence on brand image, Journal of Business Research, 62, 50-60.

Marquina-Feldman, P., Arrellano-Bahamonde, R. \& Velasquez-Bellido, I. (2014). A New Approach for measuring corporate reputation, RAE Revista de Administracao de Empresas, 1(54), 53-66.

Nguyen, N. \& Leblanc, G. (2001). Corporate image and corporate reputation in customers' retention decisions in services, Journal of Retailing and Customer Services, 8, 27736.

Nguyen, N., Yu, X., Melewer, T.C. \& Hemsley-Brown, J. (2016). Brand ambidexterity and commitment in higher education: An exploratory study, Journal of Business Research, 69, 3105-3112.

Nunnally, J. C. (1967). Psychometric Theory. New York: McGraw Hill.

Nunnally, J.C. (1978). Psychometric Theory. New York: McGraw Hill. 
Orozco, J. A. y Roca, D. (2011). Construcción de imagen de marca y reputación a través de campañas publicitarias de RSC, Sphera Publica, 11(2011), 273-289.

Pérez, A. \& Rodríguez del Bosque, I. (2014). Identidad, imagen y reputación de la empresa: integración de propuestas teóricas para una gestión exitosa, Cuadernos de Gestión, 14, 97-126.

Stern, B., Zinkhan, G. M. \& Jaju, A. (2001). Marketing images. Construct definition, measurement issues, and theory development, Marketing Theory, 1(2), 201-224.

Veloutsou, C. \& Moutinho, L. (2009). Brand relationships through brand reputation and brand tribalism, Journal of Business Research, 62(3), 314-322.

Wooldridge, J. M. (2015). Introducción a la Econometría. México: CENGAGE Learning.

Zinkhan, G. M. \& Hirschheim, R. (1992). Truth in marketing theory and research: An alternative perspective, Journal of Marketing, 56(April), 80-88. 